\title{
Ciclo de mejora en la asignatura Proyectos de la rama de Ingeniería
}

\author{
José ANTONIO VÉLEZ Godiño \\ Universidad de Sevilla \\ Departamento de Ingeniería de la \\ Construcción y Proyectos de Ingeniería \\ jvelez1@us.es \\ D.O.I.: http://dx.doi.org/10.12795/JDU.2018.i01.47 \\ Pp.: 832-849
}

\section{Resumen}

La experiencia aquí descrita se enmarca en el Curso General de Docencia Universitaria perteneciente al Programa de Formación e Innovación Docente del Profesorado (FIDOP) de la Universidad de Sevilla. El objeto de esta contribución consiste en la caracterización de una experiencia real derivada de la aplicación de la metodología basada en los ciclos de mejora a una asignatura de la rama de ingenierías. En primer lugar, se caracterizará el contexto en el que se ha desarrollado de la experiencia, procediéndose posteriormente a exponer los detalles correspondientes al diseño, aplicación y evaluación del mencionado ciclo de mejora. Este trabajo concluye la recapitulación de los hechos que han permitido la reformulación de los principios didácticos del autor.

Palabras clave: Docencia universitaria, Experimentación docente universitaria, ingeniería, proyectos, taller conceptual. 


\section{Contexto de la experiencia}

La asignatura objeto de estudio en el presente documento corresponde a "Proyectos", asignatura obligatoria cuatrimestral del Grado de Ingeniería de la Energía (3er curso), aunque también se imparte en el primer cuatrimestre en el Grado de Ingeniería de las Tecnologías Industriales (4o curso) conjuntamente con el Grado de Ingeniería Química (4o curso). La docencia de la mencionada asignatura se articula en 3 bloques (teoría, práctica y prácticas informáticas), abarcando el presente trabajo únicamente la docencia del segundo de ellos, es decir, de la parte práctica (14 sesiones semanales de 2 horas cada una). Según el plan docente vigente, mediante el bloque práctico:

"Se pretende reproducir el trabajo y la forma de llevar a cabo el desarrollo de un proyecto del ámbito de la ingeniería, con un alcance similar a un anteproyecto o proyecto básico. A los alumnos integrados en cada uno de los grupos se les asignan tareas, que se van orientando y coordinando con la participación de todos los alumnos del grupo para conseguir los objetivos del proyecto."

Por estos motivos, la docencia referida en esta aportación consiste en una suerte de juego de rol con un grupo de unos 20 alumnos, cuyo objetivo común es realizar un proyecto básico de ingeniería, el cual cambia en cada edición del curso. Por consiguiente, la docencia analizada no se fundamenta en un temario estático que impartir, sino que mediante esta asignatura se pretende abordar una serie de conceptos básicos, siempre con una vocación eminentemente práctica, con vistas a la mejora de las competencias profesionales del alumnado y lejos de los típicos desarrollos teóricos típicos de las titulaciones correspondientes a la rama de las ingenierías. El hecho anteriormente referido justifica que el profesorado que imparte el bloque práctico de la asignatura corresponda a la figura de profesor asociado, pretendiéndose de esta forma el favorecimiento de la transición universidad-empresa por parte del alumnado. A pesar de las características previamente expuestas, el mecanismo empleado tradicionalmente por el autor para impartir las sesiones formativas presenciales 
corresponde a la clase magistral, en las que el profesor actúa como un director de proyectos de ingeniería que proporciona tareas e instrucciones sobre cómo abordarlas al alumnado.

Continuando con la descripción del sistema docente, tras exponer las peculiaridades de los contenidos de la asignatura, se procede a referir que el perfil del alumnado de la asignatura analizada mediante la presente experiencia presenta el grado de madurez propio de las segundas mitades de los grados universitarios. Respecto a la implicación del alumnado en la asignatura se identifican dos claros perfiles. El primero de ellos corresponde a alumnos altamente motivados, a los que les atrae fuertemente la realización de actividades similares a las que se enfrentarán en su futura vida laboral. Por el contrario, el segundo perfil se caracteriza por un alumnado cuyo único objetivo es aprobar la asignatura consumiendo los menores recursos posibles. En el plano práctico, cabe destacar que la existencia de este segundo grupo de alumnos constituye la principal amenaza a la que se enfrenta el desarrollo de la asignatura, siendo necesario la identificación de sus integrantes y la adopción de medidas docentes para lograr la integración de estos en las actividades desarrolladas. Independientemente de los perfiles anteriormente expuestos, se ha detectado en ediciones previas del curso que la metodología empleada, la cual se fundamenta en el uso de la clase magistral, condiciona un evidente perfil pasivo del alumnado durante el desarrollo de las sesiones formativas presenciales.

Para concluir con la caracterización del sistema docente en el que se han desarrollado las acciones descritas en el presente documento, se expone que el autor del mismo posee un perfil que podría englobarse en el "modelo tecnológico de profesorado" derivado de la crítica racionalista al modelo tradicional y caracterizado por estar centrado principalmente en los contenidos impartidos (Porlán, 2017). 


\section{Diseño previo del Ciclo de Mejora Docente}

La parte práctica de la asignatura (ver sección anterior para más detalles) en la que se ha desarrollado el Ciclo de Mejora Docente (CMD) aquí descrito consta de cinco bloques temáticos: definición del proyecto, caracterización técnica, caracterización económica, cálculo de indicadores de rentabilidad y, finalmente, análisis de las conclusiones. En este punto conviene destacar que, aunque hubiese resultado más interesante aplicar la metodología posteriormente descrita a cualquiera de los otros bloques, el contenido sobre el que versa el CMD resultó completamente condicionado por el calendario académico, lo que restringió el desarrollo de este CMD a los contenidos Correspondientes a la última sesión presencial del curso, es decir, el bloque correspondiente al análisis de conclusiones. Tras acotar el temario incluido en el CMD analizado, en la Figura 1 se muestra el mapa de contenidos elaborado a tal efecto, donde se identifica el contexto del bloque analizado y sus relaciones con el resto de contenidos de la asignatura, especialmente con el bloque previo, correspondiente al cálculo de indicadores de rentabilidad. Asimismo, en el mapa de contenidos elaborado identifica los contenidos abordados durante el CMD, indicando la tipología a la que pertenece cada uno de ellos (conceptual, procedimental o actitudinal). Para concluir con el análisis del mapa de contenidos propuesto, se destaca que su elaboración ha permitido la identificación de dos contenidos como prioritarios frente al resto: en primer lugar, el concepto de riesgo en el marco del análisis de inversiones en proyectos industriales y, por otro lado, la actitud del alumnado de ingeniería frente a la redacción de reportes técnicos.

Una vez definidos los contenidos englobados en el CMD se procedió a proponer el modelo pedagógico posible. Considerando las características generales de la asignatura y las particularidades de los contenidos objeto del CMD, se optó por un modelo completamente práctico. De 
esta forma se pretende que sean los alumnos los que, a partir de los resultados de que disponen como fruto de su trabajo durante las sesiones previas del curso, vayan desarrollando las acciones necesarias para alcanzar los objetivos identificados por el profesorado. Para ello se adoptó como herramienta docente el recurso del "taller conceptual" (Finkel, 2008). Según el autor citado, la labor del profesorado no se basa en exponer sus conocimientos al alumnado, sino en aprovechar estos conocimientos para diseñar una experiencia que haga posible que el alumnado adquiera, por sus propios medios, los mismos conceptos de que dispone el profesor. A pesar de la claridad de su enfoque, esta metodología puede resultar compleja de aplicar, no sólo debido a la competencia requerida por parte del profesorado para diseñar este tipo de actividades, sino también al elevado riesgo de intervención del profesorado en las disquisiciones del alumnado, lo que conllevaría una vuelta al modelo basado en la clase magistral y en el papel completamente pasivo del alumnado.

La combinación de los contenidos mostrados en el mapa de la Figura 1 con los requisitos típicos de los talleres conceptuales permitió diseñar la secuencia de actividades que se muestra en la Tabla 1, donde también se recogen los tiempos estimados para el desarrollo de cada de ellas:

Jornadas de Formación e Innovación Docente del Profesorado | № 1 (2018) Esta obra se distribuye con la licencia Creative Commons 
INGENIERÍA

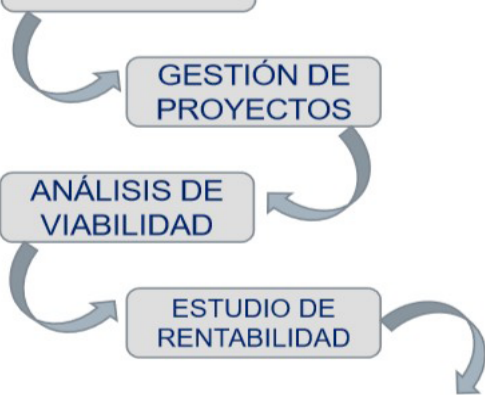

Bloque 1: Definición del proyecto.

Bloque 2: Caracterización técnica.

Bloque 3: Caracterización económica. Bloque 4: Cálculo de indicadores.

(*) Contenido básico

\section{Mapa de contenido Bloque 5: CONCLUSIONES}

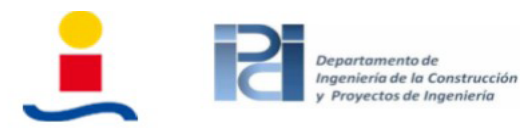

\section{VALORES}

- Racionalización del proceso de toma de decisiones

- Resolución de problemas con razonamiento crítico.

- Evaluación de la idoneidad de la información disponible.

- Análisis del impacto de las soluciones técnicas adoptadas

- Comunicación y transmisión de conocimientos.

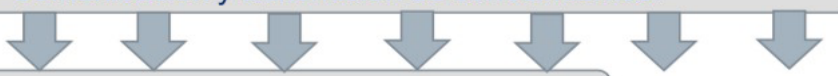

1. Análisis de conclusiones preliminares.

Actitud

\section{Bloque 5: CONCLUSIONES}

Vuelta al Bloque 4.

4. Estudio de escenarios.

5. Revisión de conclusiones. REPORTE (*).

Figura 1. Mapa de contenidos

Jornadas de Formación e Innovación Docente del Profesorado | № 1 (2018)

cc (7) $\odot$ Esta obra se distribuye con la licencia Creative Commons 
Secuencia de actividades del CMD

\section{Actividad y descripción}

ANÁLISIS PRELIMINAR

Se pretende recopilar la información disponible sobre cada proyecto y valorar de forma preliminar qué inversión sería la más rentable.

DISCUSIÓN SOBRE LA VALIDEZ DE LOS RESULTADOS ANTERIORES

Se introducirá el concepto de riesgo a través de ejemplos. Se pedirá a los alumnos que identifiquen los parámetros que conllevan un mayor riesgo de cambio.

NECESIDAD DEL ANÁLISIS DE SENSIBILIDAD

Se pedirá a los alumnos que intuyan los efectos derivados del cambio de determinados datos iniciales (riesgos). Posteriormente los alumnos deben emplear las hojas de cálculo confeccionadas durante el curso para validar sus suposiciones. Se concluirá con la necesidad de realizar análisis de sensibilidad en problemas complejos, ya que la intuición puede conducir el fallo.

ANÁLISIS DE ESCENARIOS

Se generarán los escenarios pesimista y optimista y se repetirán los cálculos de indicadores de rentabilidad según metodologías abordadas en sesiones anteriores.

DISCUSIÓN EN GRUPO

Cada grupo debe exponer los resultados correspondientes a su proyecto, lo que estará seguido de un periodo para el consenso de las conclusiones globales del proyecto (¿cuál de las 4 inversiones es más rentable?)
Temporalización

10 minutos

20 minutos

30 minutos

30 minutos

20 minutos

Para concluir con el diseño del CMD objeto de este trabajo, se procedió a confeccionar un cuestionario de exploración que, además de establecer la secuencia de preguntas necesarias para abordar las actividades recogidas en la Tabla 1, partiera del planteamiento de un "enigma" que interesase a la audiencia. Asimismo, cabe destacar que, aunque el cuestionario fue concebido con la intención de ser cumplimentado tanto con carácter previo como posterior al desarrollo de la sesión formativa 
presencial, las previamente referidas restricciones temporales padecidas forzaron que el cuestionario previo se respondiese el mismo día que la sesión presencial, lo que originó una serie de peculiaridades que serán objeto de discusión en el siguiente apartado.

\section{Aplicación del Ciclo de Mejora Docente}

Como se mencionó en el apartado previo, respecto al desarrollo real del CMD cabe destacar que la sesión comenzó con la cumplimentación del cuestionario previo. A pesar de que la realización de los cuestionarios exploratorios previo y final permitió verificar el progreso en el aprendizaje de los contenidos, la realización del cuestionario previo el mismo día que se desarrolló la sesión formativa impidió que la secuencia de actividades diseñadas se adaptase a las necesidades reales del alumnado, ya que no se dispuso de tiempo para analizar los resultados obtenidos.

Aunque el desarrollo de la sesión abarcó la totalidad de las actividades programadas (ver Tabla 1), cabe destacar que el tiempo previsto para cada una de estas actividades resultó muy distinto al realmente empleado. Resultó especialmente impactante el tiempo invertido en la actividad "necesidad del análisis de sensibilidad", mucho mayor que el previsto inicialmente debido a dificultades derivadas del esquema mental previo de la mayoría del alumnado, el cual se basaba en la capacidad de estimar resultados sin necesidad de llevar a cabo simulaciones. Por el contrario, aunque inicialmente se previó un tiempo considerable para la última actividad ("análisis de escenarios"), éste no resultó necesario, ya que el alumnado alcanzó las conclusiones previstas de forma natural y casi sin esfuerzo, como consecuencia de las actividades previas y en un tiempo menor que el esperado. 
A continuación se procede a analizar los resultados derivados de la comparación de los cuestionarios de exploración inicial y final previamente mencionados. Previamente conviene destacar que, dado que los cuestionarios fueron cumplimentados por cada uno de los grupos de trabajo establecidos para todo el curso, únicamente se dispone de cuatro respuestas. A pesar del pequeño tamaño de la muestra, se prefirió organizarlo de esta forma para favorecer el trabajo en grupo, ya que, esta metodología ha constituido una de las revelaciones derivadas de la preparación del CMD y constituye, como se expondrá más adelante, uno de los pilares básicos del modelo docente ideal pretendido. El análisis de los resultados se llevó a cabo aplicando la metodología propuesta por Ana Rivero (Porlán, 2017), la cual implica: agrupar, ordenar, analizar y, por último, establecer conclusiones (Giné y Parcerisa, 2000).

\section{Pregunta 1}

La primera pregunta constituye una singularidad del cuestionario de exploración, ya que se trata de una pregunta introductoria, sin dificultad alguna, a la que todos los grupos de trabajo respondieron bien y sin matices que posibiliten un análisis complejo, tanto en el cuestionario previo como en el final. Por este motivo no aplican más conclusiones que la correspondiente a que todos los grupos la respondieron correctamente. 


\section{Pregunta 2}

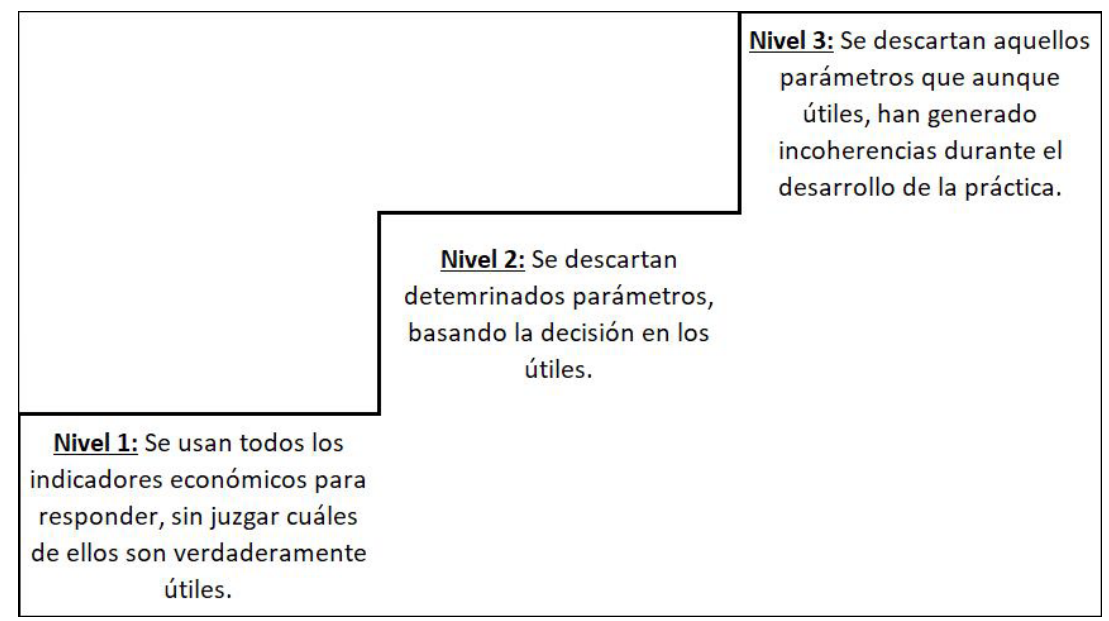

- Cuestionario inicial: Nivel 1: 3 grupos; Nivel 2: 1 grupo; Nivel 3: 0 grupos.

- Cuestionario final: Nivel 1: 0 grupos; Nivel 2: 3 grupos; Nivel 3: 1 grupo.

\section{Pregunta 3}

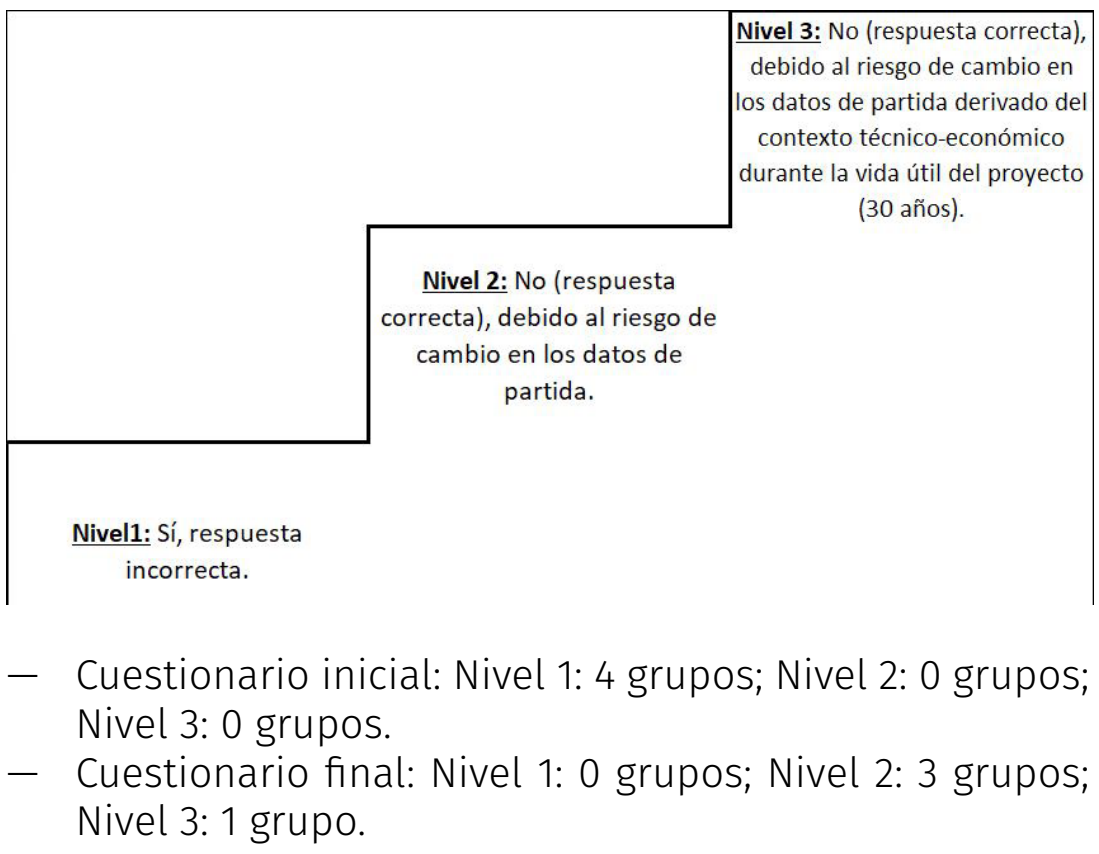

Jornadas de Formación e Innovación Docente del Profesorado | № 1 (2018) Esta obra se distribuye con la licencia Creative Commons Reconocimiento-NoComercial-SinObraDerivada Internacional (CC BY-NC-ND 4.0.) 


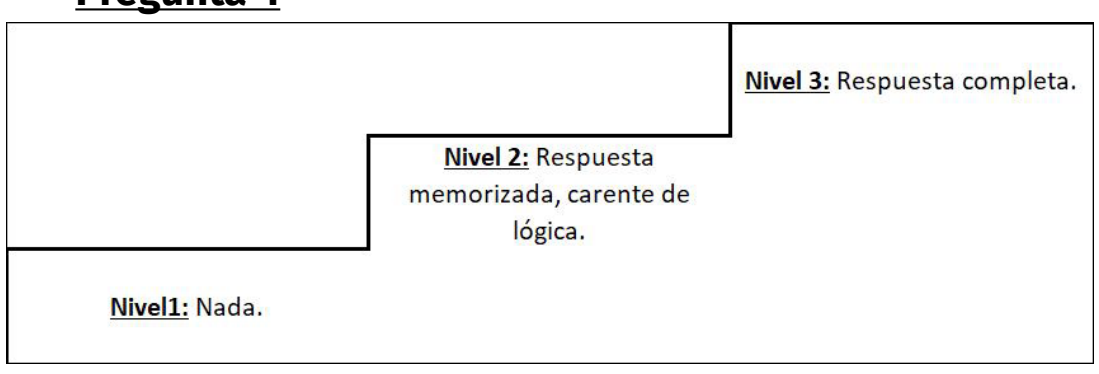

- Cuestionario inicial: Nivel 1: 4 grupos; Nivel 2: 0 grupos; Nivel 3: 0 grupos.

- Cuestionario final: Nivel 1: 0 grupos; Nivel 2: 2 grupos; Nivel 3: 2 grupos.

\section{Pregunta 5}

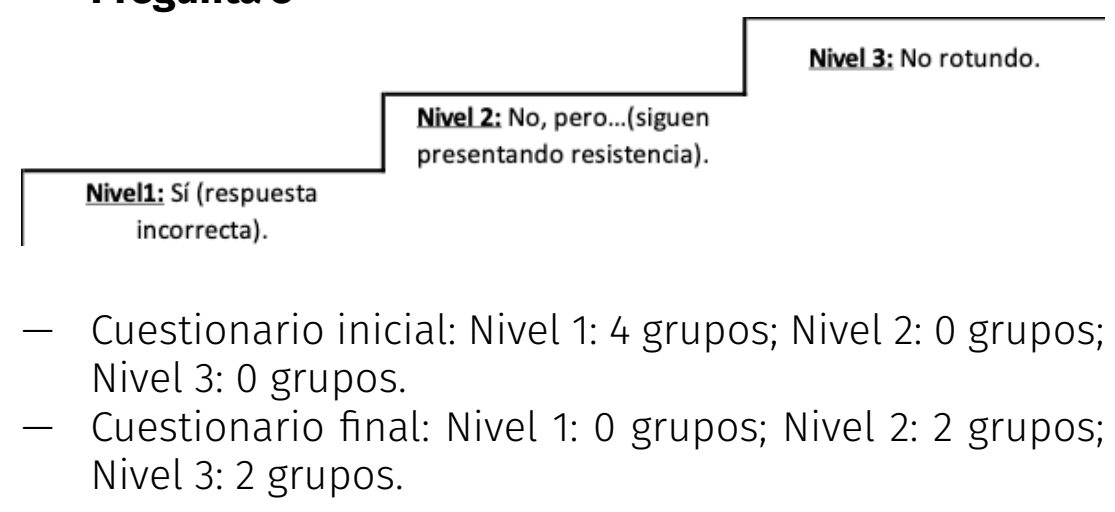

\section{Pregunta 6}

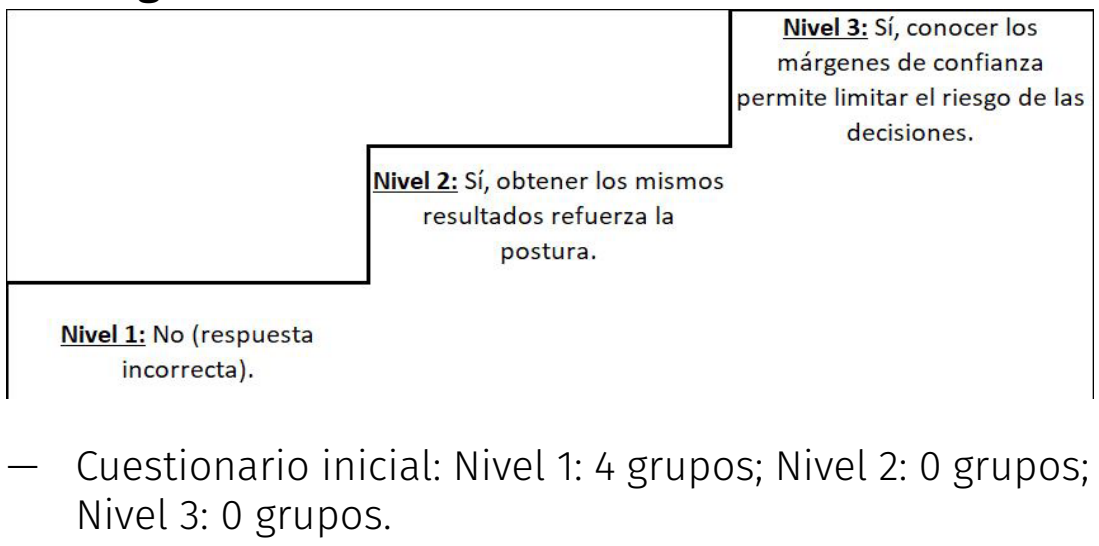

Jornadas de Formación e Innovación Docente del Profesorado | № 1 (2018)

(c) (i) (2) $\ominus$ Esta obra se distribuye con la licencia Creative Commons 
- Cuestionario final: Nivel 1: 0 grupos; Nivel 2: 2 grupos; Nivel 3: 2 grupos.

El análisis de los resultados correspondientes a las preguntas previamente expuestas, aunque correspondiente a una actividad sencilla (condicionada por el momento en el que se llevó a cabo el CMD) y con una muestra muy limitada (4 grupos de trabajo), permite obtener las siguientes conclusiones. En primer lugar, se constata que existe un progreso en el proceso de aprendizaje entre el cuestionario inicial y el final. Se atribuye este progreso a las actividades desarrolladas en clase. Asimismo, a pesar del progreso anteriormente referido, se observa que éste no es completamente homogéneo, existiendo gradaciones entre los distintos grupos evaluados. Los resultados obtenidos en el cuestionario inicial habrian permitido identificar las necesidades reales de los estudiantes, lo cual se debería haber tenido en cuenta para orientar la actividad desarrollada de haberse dispuesto de tiempo suficiente. Por último, los resultados del cuestionario final ponen de manifiesto que los conceptos cuantitativos quedan más fuertemente fijados en el alumnado que aquellos de carácter cualitativo, los cuales quedan más sujetos a interpretaciones subjetivas que dificultan su correcta asimilación.

\section{Evaluación del Ciclo de Mejora Docente}

Respecto a la respuesta del alumnado es preciso destacar que ésta fue muy positiva, concluyéndose que el alumnado es el primer interesado en abandonar las clases magistrales en las que desempeña un papel completamente pasivo, adaptándose fácilmente a modelos en los que su participación activa es clave. A pesar de esta positiva valoración, habría que revisar este comportamiento cuando el modelo se mantiene en el tiempo y se diluye el "efecto novedad" de la metodología propuesta. También es preciso destacar el resultado correspondiente al cierre 
de la sesión formativa, en el que el alumnado alcanzó las conclusiones pretendidas por sus propios medios, como consecuencia de la secuencia de acciones previamente desarrollada. Sin duda la metodología docente basada en el diseño de actividades posee un potencial muy significativo en la búsqueda de herramientas que posibiliten la adquisición por parte del alumnado de un conocimiento duradero. La discusión en grupo también merece una mención expresa, ya que típicamente había sido considerada por el autor como una herramienta que suponía más pérdida de tiempo que beneficio para el alumnado. Sin embargo, la discusión en grupo ha constituido una herramienta útil, sobre todo a la hora de resolver ciertos obstáculos en el aprendizaje, ya que unos alumnos eran capaces de resolver satisfactoriamente los problemas de otros. Este hecho resulta doblemente atractivo, ya que además de ayudar a los alumnos con problemas, permite al resto afianzar sus propias conclusiones (Bain, 2007). Para concluir con los avances, respecto a la evaluación es preciso destacar que el presente CMD constituye la primera vez que el autor ha cuantificado el proceso de aprendizaje, empleando para ello cuestionarios de exploración previo y final. Asimismo, cabe destacar el enfoque de esta evaluación, que por primera vez se planteaba como una herramienta de identificación de obstáculos (aunque en la práctica no resultó posible) y no como un elemento de calificación.

Sin embargo, se destaca que el desarrollo de este CMD se ha visto afectado por una dificultad relevante, la cual deriva del momento del curso en el que ha tenido lugar. Es decir, al llevarse a cabo en la última sesión presencial del curso no se ha dispuesto del tiempo necesario para ejecutarlo convenientemente, especialmente en relación a la realización del cuestionario previo. Se considera conveniente que se hubiese adelantado la realización del CMD en el calendario del curso, de forma que se hubiese dispuesto de margen de maniobra suficiente para adaptarse a las peculiaridades de la asignatura. Como se 
ha comentado previamente, el cuestionario previo se realizó el mismo día que la sesión formativa. Por este motivo no se dispuso de tiempo para analizar los resultados y adaptar los contenidos a las necesidades reales de los estudiantes. Este hecho impactó en la secuencia de actividades propuesta, ya que algunas tomaron mucho más tiempo del inicialmente previsto. Por último, destacar la dificultad asociada a la aparición de discusiones aparentemente sin sentido en la fase de exposición en grupo. Aunque a priori podría considerarse como una pérdida de tiempo intrínsecamente vinculada al trabajo en grupo, se considera que el balance es positivo y que la discusión, aunque aparentemente alejada de los contenidos de la sesión formativa, siempre permite abordar contenidos actitudinales. Como se ha indicará posteriormente, se considera necesario potenciar este tipo de contenidos en la formación universitaria, la cual parece estar más centrada en los contenidos conceptuales y procedimentales.

\section{Conclusión}

A continuación se resumen los aspectos abordados en este CMD que se pretende incorporar a la práctica docente habitual. En el plano de los contenidos, aunque se considera complejo abordar la totalidad de un temario, se pretende ir generando progresivamente actividades del tipo "talleres conceptuales" que sustituyan las clásicas sesiones magistrales empleadas en la actualidad. No obstante, se considera que el gran avance docente derivado del CMD descrito en este documento lo constituyen los progresos en materia de evaluación. A este respecto cabe destacar la concepción de la evaluación como una herramienta para detectar dificultades en el aprendizaje del alumnado y adaptar los contenidos proporcionados por el profesorado, no únicamente como un elemento para calificar al alumnado. Adicionalmente, se considera esencial el proceso consistente en evaluar antes (para identificar los 
esquemas mentales del alumnado) y después de las sesiones formativas (para verificar el progreso real experimentado por el alumnado).

Tras la realización del CMD aquí descrito, el autor considera imprescindible reformular su modelo metodológico previo, el cual se caracterizaba por estar centrado únicamente en los contenidos y por el papel pasivo desempeñado por el alumnado ("modelo tecnológico de profesor"). Tras la experimentación llevada a cabo durante el CMD se decide que el objetivo debe ser migrar hacia un modelo con un mayor equilibrio entre los estudiantes y los contenidos ("modelo alternativo de profesor"), en el que se identifican una serie de líneas prioritarias de actuación, las cuales se exponen a continuación. Respecto a la metodología, se pretende desplazar al profesorado del foco del proceso de aprendizaje, limitando el uso de las clases magistrales como recurso didáctico único. Con esta medida se pretende potenciar el papel del alumno, forzando su participación y la toma de conciencia en relación a su responsabilidad en su propio proceso de aprendizaje. Para ello se considera importante el uso de recursos tales como el diseño de actividades, basado en la premisa de que un aprendizaje duradero debe estar basado en experiencias y no en la memorización de conceptos; o el aula invertida, mediante la cual se pretende sacar del aula la transmisión de información, de forma que el alumnado la lleve a cabo garantizando la satisfacción de sus necesidades particulares de aprendizaje. De esta forma, el objetivo de la sesión presencial en el aula consistirá en la realización de actividades que realmente requieran la presencia simultánea del alumno y del profesor. Además, estas actividades podrían conllevar la interacción entre compañeros bajo la supervisión del profesor. Al sacar del aula la transmisión de conceptos y dedicar el tiempo principalmente a la realización de actividades, se dispone de más tiempo para que el alumnado plantee dudas al profesor. 
Para concluir se exponen los principios docentes en materia de evaluación. Como se ha mencionado con anterioridad, el gran cambio en esta materia se basa en interpretar la evaluación como una herramienta destinada a identificar obstáculos en el aprendizaje, en vez de interpretarla únicamente como una herramienta de calificación. Por ello se pretende aplicar un modelo de evaluación continua. Aunque se considera complejo debido al alto nivel de conocimiento del alumnado requerido, en la medida de lo posible se propone que esta evaluación continua sea de tipo "feed forward", ya que de esta forma se detectarian las necesidades del alumnado antes de que se produzca una desviación respecto de los objetivos establecidos. La mayor interacción entre el alumnado y el profesorado derivada del modelo metodológico de aula invertida previamente citado permite llevar a cabo una mejor evaluación como la que se propone, dado que el profesor puede analizar el desempeño de los grupos de trabajo durante el desarrollo de la sesión presencial. Además de la mencionada interacción profesor-alumno, se considera imprescindible el uso de cuestionarios de evaluación antes y después de cada bloque conceptual, de forma que sea posible evaluar las necesidades reales del alumnado y su progresión académica. Para este menester se propone el uso extensivo de "escaleras de aprendizaje".

Si bien implantar un modelo metodológico ideal resultaría excesivamente complejo a corto plazo, se pretende iniciar un proceso de reforma progresivo, con objetivos a medio plazo, de forma que desde ahora se vayan adoptando medidas encaminadas a la consecución del modelo metodológico ideal previamente descrito. 


\section{Referencias bibliográficas}

PORLÁN, R. (2017). Enseñanza universitaria. Cómo mejorarla. Madrid: Morata.

FINKEL, D. (2008). Dar clase con la boca cerrada. Valencia: Universitat de Valencia.

GINE, N. y PARCERISA, A. (2000). "Evaluación en la educación secundaria. Elementos para la reflexión y recursos para la práctica". Barcelona: Graó.

KEIN, B. (2007). Lo que hacen los mejores profesores universitarios. Valencia: Universitat de Valencia.

Jornadas de Formación e Innovación Docente del Profesorado | № 1 (2018) Esta obra se distribuye con la licencia Creative Commons 
